\title{
Computer forensics : la critique génétique et l'écriture numérique
}

Jean-Louis Lebrave

\section{(2) OpenEdition}

\section{Journals}

\section{Édition électronique}

URL : http://journals.openedition.org/genesis/633

DOI : 10.4000/genesis.633

ISSN : 2268-1590

\section{Éditeur :}

Presses universitaires de Paris Sorbonne (PUPS), Société internationale de génétique artistique littéraire et scientifique (SIGALES)

\section{Édition imprimée}

Date de publication : 30 octobre 2011

Pagination : 137-147

ISBN : 978-2-84050-804-5

ISSN : 1167-5101

\section{Référence électronique}

Jean-Louis Lebrave, "Computer forensics : la critique génétique et l'écriture numérique », Genesis [En ligne], 33 | 2011, mis en ligne le 23 octobre 2013, consulté le 30 avril 2019. URL : http:// journals.openedition.org/genesis/633; DOI : 10.4000/genesis.633 


\title{
Computer forensics : la critique génétique et l'écriture numérique
}

\author{
Jean-Louis Lebrave
}

Cet article est issu de la lecture presque simultanée de trois publications relatives aux méthodes et outils de l' informatique légale appliqués à l'écriture numérique. La première - celle qui a suscité les deux autres-est la lecture, en décembre 2010, de l'article de Thorsten Ries intitulé " "die geräte klüger als ihre besitzer". Philologische Durchblicke hinter die Schreibszene des Graphical User Interface ${ }^{1}$ ». Ries souligne l' importance pour les études textuelles contemporaines de l'ouvrage de Matthew Kirschenbaum intitulé Mechanisms. New Media and the Forensic Imagination ${ }^{2}$, ce qui m'a amené à faire une lecture critique de cet ouvrage. Enfin, j’ ai exploité le rapport auquel Lou Burnard a fait référence lors de la première séance du séminaire général 2010-2011 de l' ITEM (31 janvier 2011). Intitulé "Digital Forensics and Born-Digital Content in Cultural Heritage Collections », il a été rédigé par Matthew Kirschenbaum, Richard Ovenden et Gabriela Redwine pour le Council on Library and Information Resources des États-Unis ${ }^{3}$.

$\mathrm{L}$ 'expression anglaise « computer forensics » est généralement traduite en français par les termes suivants : « informatique légale », « investigation numérique légale », « expertise technico-légale d'ordinateurs », « inforensique », ou encore « criminalistique informatique ». Le terme anglais forensics est lui-même dérivé de l'adjectif latin forensis, puisque le forum était le théâtre public des procès à Rome, où les parties en présence présentaient leurs arguments et leurs preuves. Il désigne d'une manière générale tout ce qui a trait à la justice, et d'une manière plus spécifique l'examen scientifique des preuves susceptibles d'aider la police à résoudre les crimes. On lui fait correspondre le plus souvent en français le terme de «criminalistique ». Dès sa naissance, la critique génétique a partagé avec la science criminalistique la volonté d'étudier par des méthodes scientifiques rigoureuses les caractères matériels des documents qui constituent les dossiers génétiques, qu'il s'agisse de l'étude des supports, de celle des encres ou de l'analyse de l'écriture en tant que trace matérielle. Il est d'ailleurs très frappant de lire sous la plume de l'un des spécialistes américains d'informatique légale, à propos de la période 1999-2007, qu'il qualifie d' ' âge d'or » de sa discipline4, qu'il a été possible alors de " geler le temps et d'observer les délits en train d'être commis - et ce, même longtemps après les faits ${ }^{5} »$ : il suffit de remplacer le contexte de la scène du crime par celui du processus d'écriture pour avoir la formulation du rêve de la critique génétique.

L'informatique légale consiste en "l'utilisation de techniques spécialisées dans la collecte, l'identification, la description, la sécurisation, l'extraction, l'authentification, l'analyse, l'interprétation et l'explication de l'information numérique 6 ».

1. Thorsten Ries, «"die geräte klüger als ihre besitzer". Philologische Durchblicke hinter die Schreibszene des Graphical User Interface. Überlegungen zur digitalen Quellenphilologie, mit einer textgenetischen Studie zu Michael Speiers ausfahrt st. nazaire » [les machines plus intelligentes que ceux qui les possèdent. Coups d'œil philologiques derrière la scène de l'écriture de l'interface utilisateur. Réflexions sur la philologie des sources numériques, avec une analyse de génétique textuelle consacrée à ausfahrt st nazaire de Michael Speier], Editio, $\mathrm{n}^{\circ} 24,2010$, p. 149-199.

2. Matthew G. Kirschenbaum, Mechanisms. New Media and the Forensic Imagination, Cambridge, Mass./London, MIT Press, 2008.

3. Matthew G. Kirschenbaum, Richard Ovenden, Gabriela Redwine, Digital Forensics and Born-Digital Content in Cultural Heritage Collections, Washington, D.C., Council on Library and Information Resources, décembre 2010.

4. Comme le rappelle Thorsten Ries, cet âge d'or est mis en danger par de nouveaux procédés de stockage (celui des clés USB par exemple), mais aussi par le recours de plus en plus fréquent au nuage, qui remplace le stockage des données sur un support personnel.

5. Simson Garfinkel, « Digital forensics research: The next 10 years », Digital Investigation, $\mathrm{n}^{\circ} 7,2010$, p. 66.

6. Note d' information 04-v1 .20.doc du LERTI (Laboratoire d'expertise et de recherche de traces informatiques), 2007. 
Pourquoi et comment utiliser les méthodes et les outils de l'informatique légale dans le domaine de l'écriture numérique, et plus spécifiquement dans l'étude génétique des objets «nativement numériques » (born digital) ? L'ouvrage de Matthew Kirschenbaum, le rapport du Council on Library and Information Resources et l'article de Thorsten Ries abordent la question sous trois angles complémentaires : celui des textual studies anglo-américaines, et plus particulièrement de la bibliographie matérielle $^{7}$; celui des institutions de conservation confrontées à la multiplication des archives numériques et aux problèmes inédits que celles-ci soulèvent en matière d'archivage, de sauvegarde et de mise à disposition des communautés savantes ; celui des données que les méthodes et les outils de l'informatique légale sont susceptibles de fournir dans le cadre d'une édition critique.

On le voit, le point de vue de la critique génétique n'est pas directement pris en compte. Certes, Kirschenbaum consacre quelques lignes sommaires à ce qu'il appelle genetic editing. Mais c'est uniquement pour regretter un « effet d'aplatissement » dû à l'insertion des « variantes » « directement dans le corps du texte » au lieu qu'elles soient présentées dans un appareil critique ${ }^{8}$. Toutefois, cette définition fantaisiste est de seconde main ${ }^{9}$, et le seul exemple avancé est l'édition d'Ulysses publiée en 1984 par H.W. Gabler ${ }^{10}$, dont on ne peut pas dire qu'elle soit représentative des éditions génétiques en général. Il est dommage que Kirschenbaum n'ait pas cherché à s'informer directement sur la critique génétique. Plus intéressante est la démarche de Thorsten Ries. Celui-ci veut montrer en effet que, contrairement à une idée reçue, l'ordinateur ne met pas les généticiens au chômage par disparition des traces du processus d'écriture, et il présente un exemple convaincant d'analyse des données génétiques fournies par l'utilisation des méthodes de l'informatique légale.

Sur le plan de la critique textuelle, les références de Kirschenbaum sont pratiquement toutes américaines et anglo-saxonnes : Fredson Bowers, Jerome McGann... Il y a d'ailleurs quelque chose de choquant pour un Européen dans cette illustration d'un certain chauvinisme nordaméricain pour lequel la côte californienne et la côte atlantique des États-Unis délimitent les frontières du monde académique connu. Le plus productif de ces ancrages théoriques est constitué par la bibliographie matérielle telle qu'elle a été développée par Fredson Bowers. Kirschenbaum en transpose les principes, du support papier du livre au support magnétisé des disques durs : de même que la critique textuelle a dû dépasser la prétendue immatérialité du texte pour scruter les manuscrits et les livres dans leur matérialité, de même, on doit dénoncer la volatilité et l'« éphéméralité » supposées des documents électroniques, et donner toute sa place à la matérialité du document « nativement numérique », telle qu'elle s'inscrit sur le support magnétique des disques durs.

Tout le livre est traversé par la relation que Kirschenbaum entretient avec la critique postmoderne nord-américaine des années quatre-vingt-dix. À l'évidence, celle-ci a joué un rôle considérable dans la formation intellectuelle de l'auteur, mais son parcours l'a visiblement amené à en remettre les présupposés en cause, et l'exploration de l'informatique légale semble avoir été un puissant viatique pour y parvenir. Cette ambivalence est manifeste dans le balancement entre, d'une part, la critique, systématique, de l'immatérialité et de la volatilité des documents électroniques, telles qu'elles ont été postulées par les théoriciens américains de l'hypertexte au début des années quatrevingt-dix, et d'autre part le choix, pour illustrer la richesse de l'apport des outils développés par l'informatique légale, d'objets électroniques « postmodernes » dont les créateurs étaient très liés à ces mêmes théoriciens. Kirschenbaum analyse en effet trois documents nativement numériques : la fiction interactive Mystery House, développée en 1980

7. Voir Kirschenbaum, Mechanisms, op. cit., p. 15 : «The methodology for the book draws heavily from bibliography and textual criticism, which are the scholarly fields dedicated to the study of books as physical objects and the reconstruction and representation of texts from multiple versions and witnesses (sometimes collectively called "textual studios"). »

8. Ibid., p. 200.

9. Elles proviennent d'un ouvrage intitulé The Fluid Text: A Theory of Revision and Editing for Book and Screen, publié par John Bryant (Ann Arbor, Michigan University Press, 2002), où quelques lignes sont consacrées à l'édition génétique allemande. Pour Bryant, « the equal valorization of variant and version in geneticism tends to reduce all versions to an indiscriminate continuum of intentionality that impedes rather than furthers the study of versions » (cité d'après Kirschenbaum, Mechanisms, op. cit., p. 200).

10. J. Joyce, Ulysses. A Critical and Synoptic Edition, prepared by Hans Walter Gabler with Wolfhard Steppe and Claus Melchior, New York, London, Garland, 1984. 
pour l'ordinateur Apple II'11, l'œuvre hypertextuelle Afternoon de Michael Joyce et le logiciel Storyspace, diffusés pour la première fois en 198712 , enfin le poème Agrippa de William Gibson, apparu pour la première fois en décembre 1992 sur un Bulletin Board new-yorkais ${ }^{13}$.

Il est évident qu'il est plus facile de démontrer l'intérêt de l'informatique légale pour les études textuelles en explorant le contenu d'une disquette souple du début des années quatre-vingt qu'en analysant celui d'un disque dur d'aujourd'hui, en raison de la taille réduite de la première : comme le rappelle Kirschenbaum ${ }^{14}$, les disquettes souples de l'Apple II comportaient trente-cinq pistes contenant chacune seize secteurs contenant eux-mêmes 256 octets, soit une capacité totale de 140 kilooctets. On est très loin des gigaoctets dont dispose le moindre ordinateur portable actuel. Une analyse exhaustive du contenu d'une disquette souple est donc beaucoup plus aisée à présenter que ne le serait celle, forcément partielle, du contenu d'un disque dur. Toutefois, il est probable qu'à cette commodité matérielle vient s'ajouter chez Kirschenbaum un attrait plus nostalgique pour la disquette du logiciel Mystery House - nostalgie qu'on peut partager si on a été soi-même l'heureux possesseur d'un Apple II, et si à ce titre on s'est initié au fonctionnement du système d'exploitation et aux rudiments de la programmation en assembleur 15 .

Soulignons enfin que, même si le terme de philologie n'apparaît jamais ni sous la plume de Kirschenbaum ni sous celle de son mentor McGann, elle constitue bien le cadre conceptuel de cette approche de la textualité, sous la forme d'un implicite idéologique militant. Dans une publication en ligne récente disponible sur le site Internet de la Text Encoding Initiative, Buzzetti et McGann écrivent par exemple que l'édition critique est « centrée sur l'analyse comparative d'un ensemble de témoins documentaires » produits au cours de la «transmission textuelle16». Ces «traductions » qui sont le moyen pour les œuvres d'être transmises par un «processus de reproduction » «impliquent des transformations diverses, parmi lesquelles certaines obscurcissent et corrompent des formes textuelles antérieures ». Retrouver le texte dans sa pureté originelle, utiliser pour ce faire l' " analyse stemmatique », sélectionner les « témoins » pertinents 17 : on le voit, même non présente sous forme explicite, la philologie nourrit la démarche de ces textualistes américains, et elle le fait à travers la forme la plus archaïque des concepts philologiques. Cet ancrage traditionaliste est surprenant de la part de McGann, par ailleurs chantre de la modernité hypertextuelle depuis le milieu des années quatre-vingt-dix ${ }^{18}$.

Le conflit entre la transcendance immatérielle du texte et la matérialité contingente de ses supports est aussi au cœur de l'opposition que Kirschenbaum construit entre ce qu'il nomme la «matérialité forensique » (forensic materiality) et la matérialité formelle (formal materiality). La première est l'inscription proprement dite sur le support magnétique, celle à laquelle on peut accéder à l'aide de microscopes extrêmement puissants, et qui présente des qualités proprement stupéfiantes de résistance à l'effacement et à la destruction. Elle n'est pas différente dans sa nature des autres formes d'écriture, dont Kirschenbaum souligne d'ailleurs à plaisir qu'elles s'entrelacent aussi les unes avec les autres dans la textualité électronique. De la seconde, Kirschenbaum dit lui-même qu'elle constitue « peut-être le terme le plus difficile des deux ${ }^{19}$ ». Elle

11. Mystery House, écrit par Roberta et Ken Williams, et distribué par la compagnie Sierra Online. Voir le chapitre III du livre de Kirschenbaum : «"An Old House with Many Rooms": The Textual Forensics of Mystery_ House.dsk », p. 111-158.

12. Michael Joyce, Afternoon: a Story, First edition, Self-published, 1987 ; Third edition, Watertown, MA, Eastgate Systems, 1990 ; Fifth edition (for Windows), Watertown, MA, Eastgate Systems, 1992.

13. William Gibson, «Agrippa: a Book of the Dead », New York, Kevin Begos Publishing, 1992.

14. Kirschenbaum, Mechanisms, op. cit., p. 116.

15. Voir les deux ouvrages mentionnés par Kirschenbaum lui-même : D. Worth et P. Lechner, Beneath Apple DOS, paru en 1985, et R. Zaks, Programming the 6502, SYBEX Corp, 1981.

16. «Critical editing [...] centers in the comparative analysis of a set of documentary witnesses each of which instantiates some form or state of the work in question », Dino Buzzetti et Jerome McGann, « Electronic Textual Editing: Critical Editing in a Digital Horizon », <http://www.tei-c. org/About/Archive_new/ETE/Preview/mcgann.xml>.

17. Le modèle de l'édition critique « often postulates a single authoritative "original" state of the work. The scholar's analytic procedures are bent upon an effort to recover the text of that presumably pristine original. A key device for pursuing such a goal is stemmatic analysis [...]. A stemma of the documents means to expose, simply, which text were "copied" from which texts », ibid.

18. Voir en particulier le site Internet qu'il a consacré à Dante Gabriel Rossetti : <www.rossettiarchive.org/>.

19. «Formal materiality is perhaps the more difficult term », Kirschenbaum, Mechanisms, op. cit., p. 11. 
désigne les symboles que l'ordinateur manipule sous la forme de 0 et de 1 et les différents niveaux d'abstraction qui « entretiennent l'illusion d'immatérialité $20 »$. Certes, dans le cas des documents numériques, cette « matérialité formelle » est démultipliée par l'accumulation des couches de codages successifs, ce qui lui confère un poids particulièrement lourd. Mais en fait, elle caractérise les symboles quels qu'ils soient, et donc aussi bien toute forme d'écriture à partir du moment où, sans tenir compte de la matérialité de la substance graphique elle-même, on transforme les symboles graphiques en abstractions (on fait littéralement abstraction de la substance graphique). Or c'est bien cette abstraction qui autorise à postuler l'immatérialité du texte. Ce n'est pas le moindre paradoxe du travail de Kirschenbaum : sous couvert de combattre l'immatérialité supposée du texte numérique, la matérialité formelle se fonde en dernier ressort sur l'immatérialité du texte tout court, qu'il s'agit pour le chercheur de faire survivre aux avatars de sa transmission, mais sans se soucier de sa genèse hic et nunc au cours d'un processus de production. C'est donc en définitive une manière de couler de force toutes les traces conservées par les supports informatiques dans le moule de la textualité philologique : la «matérialité forensique » conserve les données de la «matérialité formelle », qui permet de reconstruire le stemma des versions successives du texte immatériel.

On reste médusé devant la voracité des enzymes philologiques, qui se prétendent capables de digérer sans coup férir la plus grande révolution technologique que l'écrit a connue depuis l'invention de l'imprimerie. Pour mettre l'informatique légale en œuvre, la critique génétique devra donc commencer par oublier les présupposés des textual studies américaines et revenir directement aux traces magnétisées. C'est d'ailleurs la démarche adoptée par Thorsten Ries, j'y reviendrai.

Par-delà ce vieux socle du granit philologique, les positions théoriques défendues par Kirschenbaum s'analysent en trois principes corrélés entre eux : 1) Contrairement à ce qu'ont prétendu les théoriciens américains des années quatre-vingt-dix, les objets nativement numériques ne sont ni volatils, ni éphémères, ni protéiformes ; 2) Kirschenbaum emprunte à Nick Montfort la notion d'" essentialisme écranique $^{21} \gg($ p. 34) pour dénoncer l'importance indue accordée aux interfaces utilisateurs (clavier-écran-souris) dans les travaux consacrés aux objets nativement numériques. Pour Kirschenbaum, il faut en finir avec cette dictature illégitime des utilisateurs et des usages 22 ; 3) Il convient d'aborder les documents numériques d'un autre point de vue, celui des conservateurs de bibliothèques, des chercheurs, et des spécialistes de police scientifique; on pourra ainsi apprécier à sa juste valeur la matérialité de ces documents dans sa stabilité, sa permanence, sa résistance à la destruction. S'agissant en particulier de textes, cette approche permettra notamment de suivre leur évolution à travers l'enchaînement des versions successives et des métadonnées qui leur sont attachées, car ces versions et ces métadonnées sont disséminées un peu partout dans le disque dur de l'auteur où elles ont proliféré au fur et à mesure des sauvegardes temporaires et des manipulations diverses.

Ces prises de position se fondent sur un principe non formulé, mais omniprésent : il n'y a aucune raison de s'intéresser au point de vue de celui ou celle qui produit le document nativement numérique. La genèse du texte n'est pas prise en compte, l'histoire du texte est l'histoire de sa transmission et n'est rien d'autre. C'est ce préjugé hérité de la philologie qui peut seul, en dernier ressort, fonder le rejet sans appel des caractères attribués à la «textualité électronique » de la fin des années quatre-vingt à la fin des années quatre-vingt-dix 23 : on ne voit pas pourquoi le point de vue de l'utilisateur devrait être abandonné et remplacé de manière exclusive par celui du seul chercheur ou du policier. Les deux points de vue doivent pouvoir coexister dans une théorie cohérente de la textualité. Pour le scripteur placé devant son écran, son clavier et sa souris, et dans le temps de l'écriture, le texte nativement numérique est éphémère, fluide, rhizomatique, dyna-

20. Ibid., p. 12.

21. «Screen essentialism », Kirschenbaum, Mechanisms, op. cit., p. 31 sq. 22. Il est toutefois paradoxal que la mise en cause explicite de l'utilisateur n'intervienne que dans une note consacrée à la notion d'acteur-réseau élaborée par Madeleine Akrich, Michel Callon et Bruno Latour («Introduction»), n. 31, p. 17-18: «Theories of electronic textuality have tended to privilege the user, or the text itself, rather than these and other actor-networks, which are the fruits of "science in action" and "laboratory life". »

23. Kirschenbaum, Mechanisms, op. cit., p. 166-167. 
mique. Au contraire, du point de vue, nécessairement externe, du chercheur ou du policier qui examinent après coup le support physique sur lequel le texte a été enregistré, la trace inscrite à la surface du disque dur a tous les caractères de la matérialité la plus obstinée. Seule une appréhension de la textualité comme toujours déjà-là, prête pour être transmise, c'est-à-dire corrompue, peut justifier un effacement du premier point de vue au profit du second. Si on prend en compte le sujet qui interagit avec le dispositif technique constitué par l'ordinateur et le logiciel de traitement de texte, il est bien clair que l'interaction avec les couches magnétisées du disque dur n'a aucune existence phénoménologique. Et si le fichier correspondant au texte en cours d'écriture est détruit accidentellement à cause d'une coupure de courant ou par erreur à la suite d'une fausse manœuvre de l'utilisateur, celui-ci aura bien le sentiment d'avoir irrémédiablement perdu un objet volatil. Peu lui importera dans cet instant phénoménologiquement douloureux de savoir que ses données (mais aussi toutes les métadonnées et les enregistrements temporaires de son travail) pourraient être récupérées grâce à des logiciels ultraspécialisés que leur prix prohibitif réserve à quelques bibliothèques ou laboratoires de police scientifique richement dotés en moyens. En revanche, l'accès rétrospectif à la trace matérielle est évidemment plus que précieux pour le chercheur, surtout s'il est généticien.

En résumé, Kirschenbaum a raison d'attirer l'attention sur le fait que ce qu'il appelle la «matérialité forensique » a des propriétés comparables à celles des objets imprimés, du fait même qu'il s'agit dans les deux cas d'objets matériels sur lesquels une procédure d'inscription a laissé des traces. Mais il convient d'ajouter - ce qui d'ailleurs n'affaiblit pas l'intérêt de la thèse de Kirschenbaum pour la critique génétique, au contraire - que ces propriétés ne se limitent pas aux seuls codex, ni aux «versions » successives d'un texte. Elles valent pour tout objet écrit, produit d'une inscription qui a laissé une trace sur un support. Kirschenbaum a raison aussi de souligner combien il est fécond de transposer les méthodes et les outils développés par la bibliographie matérielle pour analyser les supports matériels des documents numériques. Mais, comme le rappelle au contraire Th. Ries, ces traces n'ont pas d'existence phénoménologique pour le sujet écrivant, et elles ne peuvent être accessibles que pour un observateur intervenant après coup, une fois que l'interaction entre le support et le sujet écrivant a cessé. Il est à cet égard très caractéristique que Kirschenbaum soit littéralement fasciné à la fois par la microscopie à force magnétique, dont la résolution permet pratiquement de «voir » les molécules de substance magnétisée, et par les conseils que les services de sécurité américains donnent à qui veut détruire à coup sûr un fichier : selon la National Security Agency américaine, le meilleur moyen d'effacer le contenu d'un disque dur serait de faire passer un char dessus 24 . Aucun de ces deux dispositifs technologiques ne fait partie de l'environnement de travail d'un scripteur...

Ajoutons une dernière remarque. Dans les années quatre-vingt-dix, le terme le plus couramment utilisé pour désigner les documents produits à l'aide d'un ordinateur était l'adjectif électronique, qu'on appliquait à l'écriture, aux textes ou aux éditions. Depuis quelques années, ce terme tend à être remplacé dans l'usage par l'adjectif numérique. Kirschenbaum utilise les deux adjectifs d'une manière apparemment indifférenciée, mais les contextes dans lesquels il les emploie ne sont pas interchangeables : électronique n'intervient que dans les références aux théoriciens des années quatre-vingt-dix, alors que numérique renvoie à l'usage le plus contemporain. Il y a donc une forte corrélation entre les qualités d'éphéméralité, de fluidité, de décentrement 25 , et les qualités du courant électrique Kirschenbaum parle d'ailleurs de la «transient substance of electricity itself ». Et l'utilisateur d'un ordinateur fait couramment l'expérience concrète de ces qualités. Au contraire, tout est fait pour lui faire oublier qu'en dernier ressort, son activité consiste à inscrire des nombres sur un support magnétisé.

Moins ambitieux sur le plan théorique que l'ouvrage de Kirschenbaum, le rapport dont celui-ci est l'un des coauteurs est d'une utilisation plus immédiate pour une appré-

\footnotetext{
24. Ibid., p. 60.

25. Kirschenbaum reprend (Mechanisms, op. cit., p. 166-167) la description que Marie-Laure Ryan donne respectivement du texte imprimé et du « texte électronique »: au premier sont associés notamment les termes « durable », «linear », « centered », « stolidity », au second les termes « ephemeral », « rhizome structure », «fluidity.
} 
hension des documents nativement numériques et des archives informatiques dont les centres d'archives sont de plus en plus les destinataires. Rédigé à la demande du Council on Library and Information Resources américain par un universitaire et des représentants d'institutions de conservation, il présente un panorama très complet des problèmes que posent ces archives d'un nouveau type et des solutions que l'informatique légale est susceptible d'y apporter. Comme je l'ai indiqué plus haut, les questions soulevées et les réponses proposées ne prennent pas directement en compte la problématique génétique. Elles sont néanmoins d'un grand intérêt pour tout chercheur en génétique textuelle, parce qu'elles illustrent la richesse des matériaux numériques susceptibles d'être exploités en génétique, parce qu'elles donnent un aperçu des outils disponibles, et parce qu'elles font découvrir la complexité des problèmes posés. Le rapport est accompagné d'une bibliographie exhaustive. Il recense en outre dans plusieurs appendices les matériels et les logiciels d'informatique légale disponibles sur le marché. Enfin, il est accompagné d'encadrés qui font le point sur des questions connexes, par exemple en décrivant les ressources d'informatique légale dont se sont dotées les bibliothèques de Stanford et les Bodleian Libraries 26 .

Sans entrer dans le détail - souvent technique - du rapport, signalons quelques éléments particulièrement saillants. D'abord, on trouve une description précise des méthodes d'investigation de l'informatique légale et une analyse de la place qu'elles peuvent prendre dans le fonctionnement d'une institution d'archives, depuis le moment où les documents numériques y sont déposés jusqu'à celui où ils sont offerts à la consultation des chercheurs. Indiquons à titre d'illustration l'importance des étapes préalables à tout traitement : afin de ne pas altérer les données en travaillant directement sur le disque dur original, on doit commencer par en réaliser une « image criminalistique » (forensic image); celle-ci est créée sur un disque cible qu'on a préalablement nettoyé de toute trace susceptible d'altérer les données d'origine, en recopiant bit par bit le contenu du disque source. Il faut authentifier la fiabilité de cette image (par des techniques élaborées en cryptographie. Il faut ensuite identifier le système d'exploitation avec lequel le disque dur a été utilisé à l'origine, ce qui permettra de savoir comment les fichiers ont été stockés sur le support. Ceci conditionne la récupération des fichiers détruits intentionnellement ou accidentellement. Enfin, tous ces traitements préliminaires permettent de répertorier toutes les traces contenues dans le disque dur, en particulier les différents fichiers (intacts ou partiellement détériorés) et les métadonnées qui leur sont associées.

Parallèlement, le rapport décrit les différents types de traces qu'on peut trouver en explorant le contenu d'un disque dur à l'aide d'outils d'informatique légale. Voici par exemple quelques-unes des informations associées aux documents numériques créés avec la suite bureautique Microsoft Office - informations qui sont normalement invisibles pour l'utilisateur standard : nom du logiciel utilisé pour créer le document, identification de l'auteur du document et de ses utilisateurs, avec leur organisme de rattachement ; commentaires divers ; données extraites de bases de données ; objets insérés dans le document ; ensemble des sauvegardes rapides ajoutées à la fin du fichier ; identifiant de l'ordinateur sur lequel a été créé le document ; éléments (cellules, diapositives, textes) volontairement masqués à l'origine, puis oubliés dans le fichier par l'utilisateur qui ne les a pas supprimés ; données liées au courrier électronique, liens audio et vidéo, objets associés ; périphériques utilisés (pilotes d'imprimante); hyperliens ; identifiants des différentes sessions au cours desquelles le document a été modifié ; macros (y compris les virus) ; serveurs web, etc. ${ }^{27}$. On trouvera aussi dans l'article de Ries un exemple éclairant tiré du dossier génétique numérique du poème ausfahrt st. nazaire de Michael Speier. Les métadonnées comportent en particulier le nom du propriétaire de l'ordinateur utilisé pour créer le fichier, l'identifiant affecté à celui-ci par Word 97, la date de création, la date de la dernière sauvegarde, le nom de l'utilisateur, et la liste de dix sauvegardes « cachées » invisibles pour l'utilisateur 28 .

En deuxième lieu, le rapport aborde les problèmes épineux de conservation posés par la diversité des ordinateurs, leur évolution accélérée, et l'obsolescence des systèmes d'exploitation et des logiciels qui peuvent rendre

26. Ries donne lui aussi une synthèse des principaux éléments développés dans l'ouvrage de Kirschenbaum et dans le rapport.

27. M. Kirschenbaum, R. Ovenden, G. Redwine, Digital Forensics, op. cit., p. 45-46.

28. Ries, « die geräte Klüger... », art. cité, p. 10-11. 
rapidement la lecture des documents numériques totalement impossible. Le rapport introduit par exemple la notion de Rosetta computer, par référence à la pierre de Rosette qui permit à Champollion de déchiffrer l'écriture hiéroglyphique : sont ainsi désignés les ordinateurs à cheval sur deux technologies ou deux environnements logiciels, comme l'ont été les premiers ordinateurs encore dotés de ports série, mais déjà munis de ports USB, grâce auxquels on peut transférer des données d'un support devenu obsolète sur un support vivant. En l'absence de tels médiateurs, il est nécessaire de fabriquer les outils logiciels qui permettront de lire des formats anciens. À cette fragilité liée intrinsèquement au développement même du numérique vient s'ajouter (et ceci vient nuancer les thèses de Kirschenbaum sur la pérennité des traces numériques) la fragilité des traces elles-mêmes : le simple fait d'allumer un ordinateur ancien encore en état de marche peut entraîner l'écriture de données sur le disque dur, ce qui détruira des informations précieuses en actualisant des registres et des métadonnées ${ }^{29}$.

En troisième lieu, le rapport s'étend assez longuement sur les problèmes éthiques délicats que posent la sauvegarde et la consultation des supports numériques. Il y a là une question fondamentale par le caractère inédit des difficultés qu'elle soulève. Elle exige que « les conservateurs de bibliothèque et les archivistes remettent en question leurs conceptions de la vie privée, de l'information, des technologies, de l'accès à l'information et de l'éthique à l'ère numérique 30 ». Lorsqu'un écrivain confie ses archives papier à une bibliothèque, il peut ne pas se souvenir précisément de l'intégralité du contenu de ce qu'il donne, et avoir oublié tel ou tel détail qui figure sur l'un des documents déposés. Mais il n'a rien écrit à son insu. La situation est bien différente lorsqu'un écrivain donne ses ordinateurs et leur contenu à une bibliothèque, comme l'a fait Salman Rushdie. Tout ce qu'il a pu faire avec cet ordinateur a laissé une trace sur le disque dur. Le rapport souligne que les responsables des collections numériques dépositaires des manuscrits littéraires numériques de Salman Rushdie « pourraient avoir accès à des fragments importants de son activité personnelle sur Internet », ce qui implique, non seulement « des pratiques qui n'ont jamais eu vocation à être révélées à quiconque 31 », mais aussi des données strictement confidentielles d'ordre bancaire et médical 32 Lorsqu'il s'agit de données sensibles (archives d'hommes politiques encore en activité par exemple), il faut pouvoir assurer la sécurité du dépôt vis-à-vis de pirates exploitant les failles d'un réseau universitaire insuffisamment protégé (et convaincre les déposants de son efficacité). Il faut aussi veiller à la traçabilité des consultations des fonds et prendre les mesures adéquates pour empêcher qu'ils soient altérés par les consultations elles-mêmes. Concernant la protection de la vie privée, il faut à la fois assurer au déposant qu'elle sera satisfaisante et offrir aux chercheurs des conditions d'accès acceptables ${ }^{33}$. La responsabilité des personnels des bibliothèques est évidemment engagée, ce qui implique aussi qu'ils aient acquis la compétence technique nécessaire pour prendre les décisions pertinentes, et ce en interaction complète avec les donateurs (ceux que le rapport nomme les «créateurs de données »).

Mentionnons enfin un dernier problème, celui du coût des matériels et des logiciels d'informatique légale. La conclusion du rapport sur ce point est sans équivoque : « Le niveau d'engagement technique et organisationnel nécessaire à l'acquisition de compétences dans le domaine de l'informatique légale sera très certainement élevé [...]. Le nombre d'institutions capables de posséder ce niveau de compétence technique [...] d'une manière durable demeurera réduit. » Autrement dit, seules quelques très grandes institutions pourront assumer les coûts impliqués par la conservation des fonds nativement numériques et leur mise à disposition des chercheurs. Dans la situation actuelle de coupes drastiques dans les budgets de recherche, ce constat

29. Le problème est crucial d'un point de vue strictement criminalistique : il est nécessaire que la preuve survive à l'examen, et puisse être présentée au tribunal dans son état d'origine, sans avoir été altérée par son traitement. Le problème existe bien sûr pour les documents traditionnels écrits à la main (c'est la raison d'être de la paléographie, de la codicologie, de la diplomatique... et de l'expertise en écriture) ; néanmoins, l'écriture manuscrite apporte généralement une garantie d'authenticité qui n'a pas d'équivalent dans le monde numérique.

30. M. Kirschenbaum, R. Ovenden, G. Redwine, Digital Forensics, op. cit., p. 50.

31. Comme l'identité réelle d'utilisateurs utilisant des alias en ligne.

32. Ibid., p. 51.

33. Le rapport rappelle (p. 53) que la protection de la vie privée « constituait le principal problème » pour Salman Rushdie lorsqu'il a confié son fonds à la bibliothèque de la Emory University d'Austin, et souligne que « les bibliothécaires, les archivistes et les juristes de l'université ont travaillé pas à pas avec Rushdie pour déterminer ce qui devait rester confidentiel, et pour combien de temps ». 
est plus que préoccupant : à quelles bibliothèques les créateurs de données accepteront-ils de confier leurs archives? et ne court-on pas le risque qu'après avoir entrevu la terre promise, les chercheurs apprennent qu'elle leur est interdite faute de moyens?

Sommes-nous définitivement condamnés au pessimisme ? Le travail réalisé par Ries apporte une réponse plus qu'encourageante. À partir d'un corpus limité, et avec des outils logiciels gratuits ou peu coûteux, il montre que pour une œuvre écrite exclusivement sur ordinateur, il est possible de reconstituer un dossier génétique numérique dont la richesse est égale, sinon supérieure à celle des avant-textes manuscrits qui nous sont familiers. Il part du constat que les connaissances des spécialistes de philologie électronique sont beaucoup plus fragmentaires dans le domaine de l'informatique légale que dans celui des méthodes et des modèles d'édition numérique, et souligne que ces connaissances seraient pourtant nécessaires «pour l'élaboration d'une théorie historiquement et empiriquement fondée de l'écriture avec le médium numérique ${ }^{34}$ ». Même si, comme Kirschenbaum, Ries se réclame de la philologie et propose les premiers éléments d'une «philologie numérique des sources », il n'est pas prisonnier des présupposés philologiques les plus archaïques. D'ailleurs, lorsqu'il décrit ce qu'il nomme la «pratique philologique habituelle » avec les documents papier, il énumère des activités qui sont aussi le pain quotidien des généticiens, comme l'examen des instruments d'écriture, des supports, des tracés, ou de la mise en page.

Ries commence par rappeler la thèse selon laquelle l'écriture numérique mettra à terme les généticiens au chômage. Il l'illustre par la position prise par Derrida en 1996 et par celle, plus récente, de la textualiste néerlandaise Marita Mathijsen. À propos de la « machine à traitement de texte », Derrida considère que l'expérience de l'écriture avec un ordinateur est une « provocation » pour la critique génétique, car elle fait disparaître cette «sorte de cicatrice » que les « ratures et les surcharges » laissaient sur le papier ${ }^{35}$. Quant à elle, Marita Mathijsen se fonde sur des « conversations avec différents auteurs vivants » pour définir deux catégories d'écrivains face à l'écriture numérique : les plus âgés (l'auteur néerlandais qu'elle cite a plus de 80 ans) impriment sur papier toutes les versions intermédiaires de leur texte, effectuent leurs corrections sur cette version imprimée et n'utilisent l'ordinateur que comme une machine à écrire ; les autres (catégorie très large, puisque l'un des auteurs qu'elle cite est né en 1946, un autre en 1955, et qu'elle y adjoint « d'autres écrivains plus jeunes ») effectuent tout le travail d'écriture sur l'ordinateur, ne conservent pas les versions intermédiaires, et n'impriment que le texte définitif. Ce classement paraît très subjectif. Il n'est pas douteux que les deux types de pratiques identifiés par Mathijsen existent, mais la corrélation avec l'âge n'est certainement pas aussi tranchée qu'elle l'affirme, même s'il est raisonnable de penser qu'un scripteur qui, pendant plus de soixante ans, a travaillé avec des stylos et des machines à écrire, aura tendance à ne pas modifier en profondeur ses habitudes d'écriture. Sousjacente à la catégorisation de Mathijsen est l'hypothèse que l'écriture numérique met fin à l'écriture à programme : à propos du poète et romancier néerlandais Robert Anker, qui utilise exclusivement l'ordinateur une fois qu'il a commencé à écrire et ne garde pas de versions intermédiaires de son travail, elle affirme que « c'est la méthode de travail de quelqu'un qui ne pense plus en termes de phases de travail ». Ce jugement paraît bien hâtif et sommaire. Il mériterait d'être confronté à une enquête rigoureuse, seule à même de caractériser les usages de l'écriture numérique et leur évolution en fonction de l'âge des scripteurs.

Forte de ces interviews de quelques écrivains néerlandais, Mathijsen pronostique la disparition de la critique génétique, rendue impossible par l'écriture numérique ${ }^{36}$. À très juste titre, Ries se réjouit de souligner la fausseté de "l'hypothèse selon laquelle le processus d'écriture "ne laisse pas de traces susceptibles d'être reconstruites" avec le médium numérique » et de son corollaire qui rendrait

34. Ries, « die geräte Klüger... », art. cité, p. 150.

35. «Auparavant les ratures et les surcharges laissaient une sorte de cicatrice sur le papier ou une image visible dans la mémoire. Il y avait une résistance du temps, une épaisseur dans la durée de la rature. Désormais, tout le négatif se noie, il s'efface, il s'évapore immédiatement, parfois d'un instant à l'autre. C'est une autre expérience de la mémoire dite "immédiate" et du passage de la mémoire à l'archive. Une autre provocation pour ce qui s'appelle la "critique génétique" qui s'est développée autour des brouillons, des versions multiples, des épreuves, etc. », J. Derrida, « La machine à traitement de texte », entretien avec Béatrice et Louis Seguin paru dans la Quinzaine littéraire en août 1996 ; Papier machine, Paris, Galilée, 2001, p. 157. 
«les études génétiques impossibles 37 ». Bien que Ries insiste lui-même sur la nécessité de développer une théorie spécifique de l'écriture numérique, il renonce à explorer cette piste, et se contente d'imputer à Derrida et à Mathijsen l'« essentialisme écranique » dont Kirschenbaum postule l'existence pour en faire la critique. Ceci paraît d'autant moins justifié dans le cas de Derrida que dans son texte sur « la machine à traitement de texte », celui-ci se place très clairement du point de vue du scripteur : le fait que les corrections soient devenues invisibles pour le scripteur modifie les pratiques d'écriture. Et, comme je l'ai déjà écrit plus haut à propos de Kirschenbaum, ce qui vaut pour la trace matérielle de l'inscription sur le disque dur comme support doit être distingué soigneusement de la manière dont l'utilisateur du dispositif d'écriture numérique vit son interaction avec celui-ci. La critique génétique n'a pas seulement pour tâche d'analyser les traces magnétisées. En tant que théorie de la production écrite, il lui appartient aussi d'étudier cette interaction et d'en comprendre les effets sur le processus créateur - exactement comme elle l'a fait pour l'interaction des scripteurs avec le dispositif technologique constitué par une feuille de papier et un stylo ou un crayon.

De manière concrète, sans alourdir son texte d'un trop grand nombre de détails techniques, et en gardant toujours à l'esprit la visée génétique d'une exploration de l'avanttexte, Ries présente en particulier les principales caractéristiques des métadonnées créées par les logiciels de traitement de texte standard (la série des versions Microsoft Word 6.0 à Word 8.0 du logiciel de Microsoft Office, qui crée des fichiers avec le suffixe «.doc», et les versions plus récentes, qui recourent au format XML). Il signale différents outils courants d'exploration. Il décrit une expérience d'écriture ad hoc fabriquée pour les besoins de sa présentation et montre par des captures d'écran quelles sont les données qui ont été sauvegardées sur le disque dur, et quel est leur apport sur le plan génétique. Pour un texte de quelques lignes resté plusieurs heures ouvert sur l'ordinateur, sauvegardé quatre fois, et dans lequel le scripteur a inséré du texte à intervalles réguliers, la simple commande Undelete a recueilli pas moins de seize fichiers qui, lus avec un éditeur hexadécimal, ont permis de reconstituer la chronologie des «événements d'écriture ». Ries a même pu retrouver des données textuelles qu'il avait écrites initialement sans les sauvegarder ; elles avaient été sauvegardées par le logiciel malgré la désactivation de l'option d'enregistrement rapide. Ries conclut à juste titre de cette expérience qu'un support magnétique peut le cas échéant contenir des dossiers génétiques complets ${ }^{38}$.

Néanmoins, Ries rappelle en conclusion que, malgré la richesse de ces dossiers génétiques numériques, il manquera dans l'avant-texte ainsi retrouvé certaines des données des manuscrits de travail des écrivains : les corrections « au fil de la plume » ne sont généralement pas enregistrées, pas plus que le rythme de l'écriture - ce que Derrida appelait la « résistance » du temps. La bidimensionnalité des corrections manuscrites - ce que Ries nomme la « constellation spatiale constituée par la biffure, son remplacement et la coprésence des tracés »-est remplacée par des différences entre deux états successifs. La génétique inforensique risque donc de devoir définitivement renoncer à être une poétique des processus pour se contenter d'être une poétique des transitions entre états 39 . Il y a là un effet paradoxal : le document nativement numérique, parce que le temps n'est pris en compte que sous forme discrétisée, serait en un sens plus pauvre que le manuscrit, dans lequel toute l'information temporelle, pourtant « écrasée » dans la trace graphique (la troisième dimension, celle du temps, n'est reconstituable qu'à partir de la bidimensionnalité de

36. Marita Mathijsen, «Genetic textual editing; the end of an era », G. Mitterauer, U. Müller, M. Springeth, V. Vitzthum (éds), Was ist Textkritik? Zur Geschichte und Relevanz eines Zentralbegriffs der Editionswissenschaft, Beihefte zu Editio, n 28, 2009, p. 233-240. Concernant la critique génétique (que, comme Kirschenbaum, elle ne connaît que de seconde main), son jugement mérite d'être cité pour sa brutalité - pour ne pas dire son agressivité : «Même si la critique génétique fait partie des tendances nouvelles qui doivent encore se défendre contre les éditeurs de la collection de la Pléiade, et même si, en Allemagne aussi, elle est considérée comme innovante, je considère qu'elle est déjà dépassée parce qu'elle se fonde sur les scripteurs qui écrivent à la main, et donc sur des manières de travailler qui sont passées de mode. [...] L'étude génétique d'un texte est devenue impossible. » Elle annonce aussi une profonde mutation de l'édition critique, qui n'aura plus d'objet, puisque l'informatique fait disparaître les avant-textes et met fin aux erreurs de transmission postérieures à la première mise en circulation de l'œuvre.

37. Ries, « die geräte Klüger... », art. cité, p. 3.

38. Ibid., p. 14-18.

39. Ceci serait le triomphe des logiciels qui, comme Medite, détectent les variations entre différentes versions d'un même texte. 
la page), acquiert une présence concrète dans la substance graphique elle-même.

Il y a là un défi supplémentaire, qui n'est évoqué par aucun des trois auteurs cités ici. Il pourrait être corrigé facilement en complétant les données que les logiciels de traitement de texte enregistrent pendant l'écriture par les données en temps réel, telles qu'elles sont recueillies par des logiciels comme Scriptlog 40 ou par le mouchard Inputlog, qui fonctionne en tâche de fond en se faisant « oublier» de l'utilisateur ${ }^{41}$.

Enfin, Ries exploite l'avant-texte ainsi recueilli du poème ausfahrt st. nazaire pour une analyse génétique tout à fait convaincante. Comme il le souligne à la fin de son article, son travail a permis de montrer que les matériaux numériques - encore Ries n'a-t-il pas disposé d'une image forensique du disque dur original, ce qui l'a privé de toutes les informations contenues dans les traces résiduelles cachées - se prêtent à la fois à une critique textuelle de type philologique et à une étude génétique poussée.

Le résultat le plus tangible de ce survol est plus qu'encourageant : la critique génétique n'est en aucune manière condamnée au chômage par l'écriture numérique, au contraire. Certes, les données fournies par l'informatique légale ne se recouvrent pas avec les données des manuscrits : plus riches par certains aspects, elles sont plus pauvres pour d'autres. Mais leur abondance est impressionnante. Leur exploitation par la critique génétique pose à mes yeux deux questions. La première est d'ordre organisationnel et financier. L'informatique légale représente une branche hautement spécialisée de l'informatique, dont la maîtrise suppose des compétences très étendues. On voit mal comment on pourrait demander aux chercheurs en génétique, le plus souvent de formation littéraire, d'acquérir de telles compétences. On pourrait en revanche imaginer que les laboratoires de recherche et les institutions de conservation avec lesquelles ils travaillent puissent se doter d'informaticiens spécialistes capables d'extraire des disques durs les données dont ils ont besoin en respectant les contraintes, notamment éthiques, liées à cette fouille de données très particulière. Le rapport rédigé par Kirschenbaum et al. montre la voie dans ce sens. Mais il insiste aussi sur le coût élevé que représentent l'archivage et la consultation de ces données nativement numériques.
Contrairement à l'affirmation péremptoire de Mathijsen, la critique génétique n'est pas menacée par la disparition des avant-textes. L'écriture numérique les fait au contraire proliférer. Il serait catastrophique que, faute de moyens matériels, cette richesse devienne encore plus inaccessible qu'un fonds manuscrit non catalogué dans une petite bibliothèque de province.

Soyons optimiste, et supposons que ce problème trouvera une solution satisfaisante. Se pose alors une question proprement scientifique. Il importe de ne pas se laisser enfermer dans une appréhension restrictive de la trace magnétique comme trace d'une histoire des versions du texte. Parallèlement au point de vue du chercheur - dans ce cas, il tend à se confondre avec celui de l'ingénieur de la police scientifique - qui examine la trace après coup, il convient que soit exploré aussi le point de vue de l'utilisateur, scripteur ou écrivain, pour lequel les logiciels d'informatique légale ne seront jamais des outils d'écriture. Il faut préserver l'opposition entre, d'un côté, la volatilité et l'éphéméralité électroniques, qui sont les qualités des objets nativement numériques pour l'écrivain, et de l'autre, la matérialité résistante de la trace magnétique numérique, car ce sont les deux angles d'attaque complémentaires d'une exploration de la genèse des textes. Comment écriton ? Que nous apprend la trace écrite sur le processus d'écriture ? C'est bien en effet par ces deux questions que la critique génétique se définit depuis sa naissance.

Paris, janvier-avril 2011

40. Pour une présentation de Scriptlog, voir S. Strömqvist, « Une approche expérimentale du processus d'écriture : l'enregistrement de la frappe au clavier », Genesis, n² 27, 2006, p. 45-57.

41. Mariëlle Leijten et Luuk Van Waes, « Inputlog: New Perspectives on the Logging of On-Line Writing », K.P.H. Sullivan \& E. Lindgren (éds), Studies in Writing, vol. XVIII, « Computer Key-Stroke Logging and Writing: Methods and Applications », Oxford, Elsevier, 2006, p. 73-94. 
JEAN-LOUis LEBRAVE est directeur de recherche émérite à l'ITEM (CNRS-ENS). Il a travaillé notamment sur les manuscrits de Heine, de Proust, de Flaubert, de Barthes, de Vitez. Outre des publications sur la théorie génétique, il s'est intéressé à l'hypertexte («Hypertextes - Mémoires - Écriture », Genesis, n 5, 1994), aux traitements informatiques des données manuscrites et à l’incidence des technologies de l'information sur l'écriture (Texte et ordinateur : les mutations du lire-écrire, Espace européen, 1991, avec J. Anis ; « Retour vers le futur. Supports anciens et modernes de la connaissance », Diogène, n 196, 2001, avec J.-G. Ganascia). Par ailleurs, il s'est attaché à l'analyse des dimensions psycholinguistiques de la critique génétique.

Jean-Louis Lebrave, jean-louis@lebrave.name

Résumés

\section{Computer forensics : la critique génétique et l'écriture numérique}

L'informatique légale (computer forensics) nous révèle que, contrairement à une crainte souvent formulée, l'abondance et la robustesse des traces que le processus créateur laisse dans l'écriture numérique sont spectaculaires. À partir de trois publications récentes, on explore cette nouvelle forme de codicologie numérique. Contrairement à ce qu'affirme un représentant contemporain de la critique textuelle américaine comme Matthew Kirschenbaum, l'enjeu dépasse de beaucoup l'exploration philologique traditionnelle des versions successives d'un texte. D'une part, la conservation et l'exploitation des traces numériques posent des problèmes inédits aux responsables des bibliothèques et des archives. D'autre part et surtout, l'étude que Thorsten Ries a menée sur des matériaux génétiques numériques dévoile la spectaculaire richesse de ceux-ci. Loin d'être condamnée à disparaitre par l'avènement du tout numérique, la critique génétique a de beaux jours devant elle, pour peu qu'elle apprenne à recourir aux outils et aux méthodes de l'informatique légale.

Computer forensics reveal that, contrary to an often expressed concern, the abundance and vigor of the traces the creative process leaves in digital writing are spectacular. With three recent publications we will explore a new kind of digital codicology. Contrary to the claims of Matthew Kirschenbaum - a contemporary representative of American textual criticism -, the issue goes far beyond the traditional philological investigation of a text's successive versions. On the one hand, the conservation and exploitation of digital traces raise new problems for librarians and archivists. But on the other, more significantly, Thorsten Ries' study of digital genetic material reveals its spectacular wealth. Far from being doomed to disappear, owing to the advent of the all-digital, genetic criticism still has a future, if it learns to use computer forensics' tools and methods.

Die computergestützte Forensik zeigt uns, entgegen oft geäußerter Bedenken, dass die Vielzahl und Robustheit der Spuren, die der kreative Prozess beim digitalen Schreiben hinterlässt, spektakulär sind. Ausgehend von drei vor kurzem erschienenen Publikationen, soll diese neue Form der digitalen Kodikologie untersucht werden. Entgegen der Ansicht eines Vertreters der amerikanischen Textkritik wie Matthew Kirschenbaum, geht die Herausforderung weit über die traditionelle philologische Untersuchung aufeinanderfolgender Textversionen hinaus. Zum einen stellt die Konservierung und Auswertung der digitalen Spuren die Bibliotheken und Archive vor noch nie dagewesene Probleme. Zum anderen hat insbesondere die von Thorsten Ries durchgeführte Studie über digitale genetische Materialien deren spektakuläre Reichhaltigkeit aufgezeigt. Weit davon entfernt wegen der Inthronisierung der digitalen Welt auszusterben, sieht die critique génétique guten Zeiten entgegen, wüsste sie sich nur der Software und der Methoden der forensischen Informatik zu bedienen.
La informática forense (computer forensics) nos revela que, contrariamente a un temor que se manifiesta a menudo, la abundancia y la robustez de las huellas que el proceso creador deja en la escritura digital son espectaculares. A partir de tres publicaciones recientes, exploramos esta nueva modalidad de codicología digital. Contrariamente a lo que afirma un representante contemporáneo de la crítica textual norteamericana como Matthew Kirschenbaum, los propósitos van mucho más allá de la tradicional exploración filológica de las versiones sucesivas de un texto. Por una parte, la conservación y la explotación de las huellas digitales plantean problemas inéditos a los responsables de bibliotecas y archivos. Por otra parte, y sobre todo, el estudio realizado por Thorsten Ries sobre los materiales genéticos digitales ha puesto en evidencia la espectacular riqueza de los mismos. La crítica genética no está condenada a desaparecer ahogada por las prácticas digitales, sino que, por el contrario, tiene un papel importante que jugar, siempre y cuando acepte recurrir a las herramientas y los métodos de la informática forense.

L'informatica legale (computer forensics) ci mostra che, contrariamente ai timori diffusi, l'abbondanza e la sostanza delle tracce che il processo creativo lascia nella scrittura su computer è spettacolare. A partire da tre recenti pubblicazioni, si esplora questa nuova forma di codicologia informatica. Contrariamente a quanto afferma un rappresentante contemporaneo della critica testuale americana, Matthew Kirschenbaum, la posta in gioco è ben superiore all'esplorazione filologica tradizionale delle versioni successive di un testo. Da una parte, la conservazione e l'analisi delle tracce informatiche pongono problemi inediti ai responsabili di biblioteche e archivi. Dall'altra, soprattutto, lo studio di Thorsten Ries su materiali genetici informatici svela la loro spettacolare ricchezza. Ben lontana dall'essere condannata a sparire dall'avvento dell'informatizzazione della scrittura, la critica genetica ha un bel futuro davanti a sé: è sufficiente che impari a utilizzare gli strumenti e i metodi dell'informatica legale.

A informática forense (computer forensics) revela que, contrariamente a um receio frequente, a abundância e o vigor dos rastos que o processo criador deixa na escrita digital são espectaculares. A partir de três publicações recentes, explora-se essa nova forma de codicologia digital. Contrariamente ao que afirma um representante contemporâneo da crítica textual americana, Matthew Kirschenbaum, está em jogo muito mais que a exploração filológica tradicional das versões sucessivas de um texto. Por um lado, a conservação e a exploração dos registos digitais colocam problemas novos aos responsáveis das bibliotecas e arquivos. Por outro lado, e principalmente, o estudo que Thorsten Ries efectuou sobre materiais genéticos digitais revela a sua espectacular riqueza. Longe de estar condenada a desaparecer pelo advento do mundo digital, a crítica genética tem um risonho futuro pela frente, desde que aprenda a recorrer aos instrumentos e aos métodos da informática forense. 